\title{
BIBLIOGRAPHIE
}

[1] Ch. Porcher. Le Lait, 1929: 88, $81 ; \mathbf{8 9}, 962 ; 1930: \mathbf{9 4 ,} 417$.

[2] Alais. Le Lait, $1950: 30,349$.

[3] OrLa-Jensen. Le Lait, 1924: p. 468 ; p. 537.

[4] Orla-Jensen, V. C. Otte, A. Snog-KJaer, Mémoires de l'Agadémie Royale de Danemark, 1936, t. VI.

[5] R. Vimille. Thèse, Lille, 1936.

\section{CONTRIBUTION AU CONTROLE BACTÉRIOLOGIQUE DU LAIT}

\section{par \\ Dr COSTAS G. MACRIS et COSTAS G. TZIVANOPOULOS}

\section{A. Action réductrice des microbes}

1. Le nombre de microbes et le degré de contamination du lait. - L'examen des aliments du point de vue hygéinique, particulièrement ceux d'origine animale, est d'une plus grande importance pour la santé publique, que le contrôle officiel des marchés se bornant à constater les falsifications ou simplement les altérations, visibles à l'œil nu, des aliments.

Le lait, produit biologique d'une importance capitale pour la nutrition de l'homme, est un terrain exceptionnel pour le développement et la multiplication des microorganismes, surtout pendant les saisons chaudes. C'est ainsi que cet aliment important devient souvent transmetteur de maladies, notamment chez le nourrisson et l'enfant.

Le nombre de tels microbes, chaque fois contenu par le lait, détermine son degré de pureté et, réciproquement, son degré de contamination.

La détermination du nombre de microbes et, notamment, celui des colibacilles, présente un grand intérêt du point de vue du contrôle d'hygiène du lait. Ces microbes qui, normalement, n'auraient pas dû exister dans le lait, constituent un critérium important du degré de contamination de celui-ci, et la source principale des colibacilles est représentée par les matières fécales des animaux et de l'homme, lesquelles, par ailleurs, peuvent également contenir bien d'autres microbes pathogènes.

Le contrôle du nombre de colibacilles contenu dans le lait cru et, plus spécialement, dans le lait pasteurisé, doit donc être tenu pour indispensable. Ce n'est que dans les pays ne possédant pas de contrôle d'hygiène, systématiquement organisé, des aliments, qu'il est permis d'offrir à la consommation du lait pasteurisé, sans que cer- 
taines restrictions sanitaires élémentaires aient été préalablement appliquées.

L'abondance des colibacilles dans le lait cru dénote la non observance des dispositions sanitaires requises au cours de la mulsion et de la récolte du lait, ou bien la contamination de celui-ci au cours de son transport ou son transvasement, ou bien son stockage défectueux.

La présence de colibacilles nombreux dans le lait pasteurisé constitue une preuve du fonetionnement défectueux des appareils de chauffage, d'une mauvaise pasteurisation, d'une mauvaise conservation, d'une utilisation de récipients malpropres, etc.

Le contrôle d'hygiène du lait pasteurisé est d'une très grande importance, car la plupart s'imaginent, à tort, que tout lait pasteurisé est sain, exempt de microorganismes, et qu'il peut être consommé sans danger.

Néanmoins, en l'absence de tout contrôle et offert à la consommation sans la garantie du producteur responsable, il devient dangereux, plus particulièrement pour le nourrisson et l'enfant auxquels, dans lé but d'éviter toute perte de vitamines due à la cuisson, il est fréquemment donné cru.

En Allemagne, depuis février 1932, le contrôle d'hygiène próvoit qu'un bon lait pasteurisé ne doit pas contenir plus de 150.000 germes microbiens par centimètre cube, ni plus de 30 colibacilles. On procède au contrôle en cultivant pendant dix-huit heures à l'étuve, $0 \mathrm{~cm}^{3} 1$ de lait sur de la gélose additionnée de bleu de bromothymol et de tripaflavine, ensuite on dénombre les colonies jaunâtres qui se sont développées.

Aux Etats-Unis, le contrôle d'hygiène exige que le lait cru garanti soit exempt de colibacilles. Par contre le règlement ne prévoit rien à l'égard du lait ordinaire ni des diverses qualités de lait pasteurisé.

En Angleterre, le contrôle d'hygiène prescrit que le litre de lait pasteurisé de première qualité ne doit pas contenir plus de 10.000 colibacilles. Quant aux autres qualités pasteurisées, le nombre de colibacilles ne doit pas dépasser le chiffre de 100.000 le litre.

En France, le décret du 26 avril 1939 prévoit qu'un centimètre cube prélevé sur du lait pasteurisé garanti, ne doit contenir aucun bacille des groupes Escherichia et Aerobacter. Ce décret ne mentionne rien quant au nombre toléré de ces microbes dans le lait pasteurisé ordinaire. Le décret du 28 avril 1939, relatif au contrôle d'hygiène du lait cru, ne prévoit également rien au sujet du nombre de colibacilles.

Selon le Code grec, et les ordonnances relatives aux aliments, boissons et objets d'usage commun, Athènes, 1941 [1], il est inter- 
dit de mettre en vente du lait dont le degré de contamination déterminé par son pouvoir de décomposition est tel que la décoloration du bleu de méthylène a lieu en moins de deux heures.

Le Code grec distingue plusieurs qualités de lait :

a) Bonne qualité. La décoloration du bleu de méthylène a lieu au bout de 5 heures et demie à 10 heures.

Un centimètre eube de cette qualité de lait peut contenir jusqu'à 500.000 microorganismes.

b) Qualité médiocre. La décoloration a lieu au bout de 2 heures à 5 heures et de mie.

Nombre de microorganismes par centimètre cube, de 500.000 à 4.000 .000 .

c) Très mauvaise qualité. Décoloration au bout de 20 minutes.

Nombre de microorganismes par centimètre eube, de quatre à vingt millions.

d) Qualité exceptionnellement mauvaise. Décoloration au cours des premières vingt minutes.

Nombre de microorganismes par centimètre cube, plus de vingt millions.

A lire ce qui précède, on peut aisément se rendre compte de l'importance universellement accordée au contrôle des qualités de lait susceptibles d'être consommées sans cuisson, telles que le lait cru garanti et le lait pasteurisé.

\section{Les diastases microbiennes et le contrôle d'hygiène}

du lait. - La méthode la plus commode et la plus courante pour déterminer le degré de contamination du lait, est celle qui se base sur l'action altérante des produits du fonctionnement biologique des microorganismes du lait, c'est-à-dire, au cours de l'action de la réductase et de la catalase.

La réductase mierobienne présente une action très différente de celle de la réductase cellulaire du lait.

La technique de la réductase constitue un moyen aisé de contrôle microbien du lait, parce que la décoloration du bleu de méthylène a lieu d'autant plus vite que le nombre des microbes existant dans le lait est plus grand.

La capacité d'activité de la catalase est basée sur la vitesse avec laquelle se décompose le peroxyde d'hydrogène par le lait.

En Europe, et, plus particulièrement, en Suède et au Danemark, la méthode de la réductase est très en faveur, ses résultats présentant un facteur d'estimation dans l'appréciation de la qualité du lait.

En réalité, la méthode de la réductase ne peut être considérée 
comme donnant des résultats absolument certains, car des facteurs autres que le nombre, plus ou moins important, de mierobes, peuvent également contribuer à une rapide décoloration du bleu de méthylène.

3. Action dénitrifiante des microbes. - Nombreux sont les microbes en état de produire la dénitrification des matières azotées. Cette propriété n'est pas identique chez tous les microbes dénitrifiants. Elle varie, tant par son degré d'intensité, que par la nature même de son action. Au cours de cette action de décomposition, les combinaisons nitriques peuvent être transformées en nitrites, en ammoniaque, en oxydes d'azote, leur décomposition pouvant même être poussée jusqu'à libération d'azote libre.

C'est Shonnbern [2], qui le premier (1864) s'est occupé de l'action dénitrifiante des microbes. Plus tard (1882) Röнmann [3] constata que les fonds d'égout ont une propriété puissante de décomposer les nitrates. GuYon et PETIT [4]. parvinrent à découvrir dans les eaux stagnantes des égouts un microbe anaérobie doué d'une action puissamment dénitrifiante. MAASSEN [5] cultiva, dans une solution de nitrate de soude additionnée de peptone, 106 espèces différentes de bacilles tirées du sol. Il constata que les deux tiers, environ, des microbes cultivés, étaient cause de décompositions n'atteignant que le stade azoteux ; l'autre tiers poussait la décomposition jusqu'à formation d'ammoniaque et deux espèces de microbes parvenaient seules à pousser la décomposition jusqu'à la production d'azote non combiné.

Les principaux microbes doués de propriétés dénitrifiantes, selon leur degré d'intensité, sont les suivants : $B$. Coli, B. vulgare, $B$. prodigiosus, B. putridum, B. fluorescens, B. pyocyaneum, B. subtilis, B. lactis aerogenes, B. mucoides, le staphylocoque, etc. Le colibacille, notamment celui de l'homme, a un pouvoir particulièrement grand de transformer les nitrates en nitrites.

Le streptocoque, le bacille de la tuberculose et le gonocoque n'exercent aucune action dénitrifiante.

\section{B. Méthode de la nitroréductase}

1. Avantages et inconvénients de la méthode. - Il ressort de notre bref exposé que les colibacilles sont les microbes les plus courants constituant le prineipal agent de contamination du lait. Nous venons de voir, par ailleurs, que ces bacilles sont doués d'un pouvoir dénitrifiant intense qui se manifeste par la décomposition des nitrates en nitrites. Selon notre opinion, cette action du colibacille ainsi que celle des quelques autres bacilles du groupe Coli, etc., pourrait servir de critérium important dans la détermination 
du degré de pureté du lait. Partis de cette idée, nous nous sommes efforcés de créer une technique simple et utile en tant que constituant une méthode rapide et sûre de contrôle d'hygiène du lait.

La méthode que nous proposons dans notre présente communication, nous l'appelerons "méthode de la nitroréductase ", car elle est basée sur l'action dénitrifiante des microbes du lait. Cette action biocatalytique est d'une intensité proportionnelle au nombre de microbes dénitrifiants. Lorsque nous déterminons done, par périodes de temps, la quantité de nitrites formée, à la température de $37.40^{\circ} \mathrm{C}$, dans le lait examiné, après l'avoir convenablement dilué avee une solution d'un sel nitrique, nous pouvons déterminer le degré de contamination d'un lait, par le colibacille et, d'une manière générale, par tous germes microbiens ayant la propriété de réduire les nitrates en nitrites.

Nous pensons que, comparée à la méthode de la réductase, celle de la " nitroréductase » donne des résultats plus sûrs ear, tandis que, dans la réductase, la décoloration du bleu de méthylêne peut être également due à d'autres agents, dans celle de la nitroréductase la décomposition des nitrates est exclusivement due à l'action microbienne.

On pourrait objecter, qu'en procédant au contrôle d'hygiéne du lait, par la méthode de la nitroréductase, certains microbes importants, non doués d'une action dénitrifiante, tels que le streptocoque et le bacille de la tuberculose, ne sont pas pris en considération. Néanmoins, nous ne croyons pas que eette objection ait, en l'occurrence, une importance particulière, ni qu'elle puisse constituer une critique sérieuse à l'égard de la valeur de la nitroréductase.

Nous savons qu'un grand nombre de méthodes sont préconisées dans le contrôle microbien du lait, spécialement du lait pasteurisé. Toutefois, aucune des méthodes existantes ne peut être tenue pour parfaite. Le bacille de la tuberculose est plutôt rare, sa culture est particulièrement difficile et il est l'un des microbes asporulés des plus résistants, de sorte que la constatation de sa présence ne peut servir de critérium chaque fois qu'il s'agit de déterminer si la pasteurisation du lait a été suffisamment poussée.

C'est la raison pour laquelle on se sert fréquemment, pour ce genre de contrôle, du colibacille qui est moins résistant à la chaleur que le Mycobact. tuberculosis. La recherche du colibacille a été jugée comme pouvant efficacement servir au contrôle d'hygiène du lait pasteurisé. Cependant, nous ferons observer que la colimétrie, qui est la méthode la plus usitée, ne fournit qu'un indice dans la détermination du degré de pureté du lait cru ou pasteurisé, encore que cette méthode, basée sur une estimation approximative 
du nombre de colibacilles, soit plus longue et plus compliquée que celle de la réductase.

2. Technique de la nitroréduction. - Nous donnerons dans cette communication préliminaire la méthode que nous avons essayée pour le contrôle d'hygiène des laits crus et pasteurisés de diverses provenances.

\section{A. Instruments et produits nécessaires}

1. Sel nitrate en solution stéritisée. On prépare cette solution avec $0 \mathrm{gr} .9$ de chlorure de sodium et $0 \mathrm{gr}$. 1 de nitrate de potasse dissous dans $100 \mathrm{~cm}^{3}$ d'eau distillée. La solution est stérilisée par ébullition dans un Erlenmeyer. On recouvre ce dernier avec de l'ouate non hydrophyle.

2. Réactif Illosvay pour la recherche des nitrites. Ce réactif se compose de deux solutions :

a) 0 gr. 5 d'acide sulfanilique dissous dans $150 \mathrm{~cm}^{3}$ d'acide acétique dilué.

b) 0 gr. 2 de a.naphtylamine dissous dans $20 \mathrm{~cm}^{3}$ d'eau chaude. La solution est filtrée à travers un petit filtre dans $75 \mathrm{~cm}^{3} \mathrm{~d}$ 'acide acétique dilué.

Le réactif Il'o svay est d'une sensibilité de $1: 5.000 .000$. Il doit être conservé dans deux flacons opaques, sinon la solution de naphtylamine s'altère. Il peut être également utilisé sous forme d'unique solution, il suffit de filtrer la solution de naphtylamine dans la solution acétique d'acide sulfanilique. Nous avons constaté que l'on peut augmenter la sensibilité du réactif aux nitrites, en utilisant séparément une solution à $10 \%$ de a-naphtylamine et une solution saturée de benzidine dans de l'acide acétique.

Pour la recherche des nitrites, on peut également utiliser une solution aqueuse de sulfamidés. Presque tous les sulfamidés, au contact des nitrites donnent la même coloration rosée caractéristique obtenue avec le réactif Illosvay au contact des solutions très diluées de nitrites.

Lorsque l'on utilise le réactif Illosvay en deux solutions séparées, on verse 2-3 gouttes de la solution de a-naphtylamine et ensuite 2-3 gouttes de la solution d'acide sulfanilique. Lorsqu'on utilise ce réactif sous forme d'une seule et unique solution, on verse 5-6 gouttes de réactif dans le lait à examiner.

A part les réactifs que nous venons de mentionner, la méthode de la nitroréductase exige, comme matériel de laboratoire, des éprouvettes stériles et une étuve ou un bain-marie susceptible de maintenir une température constante de $37-40^{\circ} \mathrm{C}$. 


\section{B. Mise en pratique de la méthode}

On prend 5 tubes à essais. Dans chacun d'eux on verse $10 \mathrm{~cm}^{3}$ de solution nitrate. A l'aide d'une pipette, on introduit attentivement et rapidement (afin de ne pas contaminer le liquide) $0 \mathrm{~cm}^{\mathbf{3}} \mathbf{5}$ de lait dans le premier tube à essais, $0 \mathrm{~cm}^{3} 4$ dans le second, $0 \mathrm{~cm}^{3} 3$ dans le troisième, $0 \mathrm{~cm}^{3} 2$ dans le quatrième et $0 \mathrm{~cm}^{3} 1$ dans le cinquième. On les bouche tous avec un tampon.

Ces tubes à essais sont chauffés à la température de $37-40^{\circ} \mathrm{C}$. à l'étuve ou au bain-marie, et l'on note l'heure exacte à laquelle l'expérience a été commencée.

Après un quart d'heure de chauffage, à cette température, a lieu le premier contrôle de la réduction du nitrate. On procède aux contrôles suivants, d'heure en heure. Lorsque le lait est extrêmement contaminé, la réaction décelant le nitrite apparaît fortement positive au cours de la première heure.

Pour la recherche des nitrites on transvase successivement et soigneusement dans des tubes propres, environ $2 \mathrm{~cm}^{3} \mathrm{du}$ lait contenu dans les tubes chauffés à l'étuve et l'on y ajoute le réactif Illosvay, c'est-à-dire, dans chacun d'eux 2-3 gouttes de la solution d'acide sulfanilique et $2-3$ gouttes de la solution de a-napthylamine, ou bien, 2-3 gouttes de solution de benzidine et ensuite 2-3 gouttes de naphtylamine.

Si la réduction du nitrate est fortıment avancée, le lait des tubes d'essai, après écoulement de 1-2 minutes, acquiert une coloration qui peut aller du rose pâle au rouge cerise. En y ajoutant la benzidine, la couleur devient plus vive et tend vers le violet. Lorsque la quantité de nitrite contenue est de l'ordre de 0 mgr. 1, nous devons faire remarquer que le réactif Illosvay est défaillant, et l'on doit user de la solution de benzidine suivie de celle de naphtylamine.

\section{Critique des résultats}

Un lait cru ou pasteurisé, très pur, donne une réaction négative ou très faiblement positive. C'est-à-dire, qu'en l'espace de quelques minutes, une légère coloration rosée peut se manifester, qui correspond à une quantité de nitrite de l'ordre de 1 :10.000.000.

A ce stade, la réaction reste la même pendant une période de plusieurs heures. Ce n'est qu'après écoulement de dix-huit heures que celle-ci devient fortement positive (couleur rouge cerise).

Des laits considérés, selon le Code grec, de mauvaise qualité, donnent une réaction positive après une heure et demie de chauffage à l'étuve.

Enfin, des laits très impurs, c'est-à-dire, très riches en coli- 
bacilles et autres bacilles dénitrifiants, donnent de fortes réactions positives au cours de la première heure de chauffage à l'étuve.

Nous avons constaté que, dans la méthode de la nitroréductase, l'usage de laits très dilués, tels que ceux employés dans nos expériences d'essai, donnent de meilleurs résultats. Lorsque le lait n'est pas suffisamment dilué dans la solution de nitrate, son oxydation pourrait entraver le cours de la réduction. Il se pourrait même que celle-ci contribue à la réoxydation des légères quantités de nitrite, initialement formées, par l'action des microbes, fortement décomposante au début.

Nous continuons systématiquement nos recherches, dont nous espérons pouvoir donner les résultats dans une prochaine étude.

\section{Conclusion}

L'action des différents microbes pendant la période de leur fonctionnement biologique, fournit un terrain de recherches extrêmement intéressant.

L'une de ces formes d'énergie est l'action dénitrifiante possédée par des microbes variés et, en particulier, par le colibacille.

Dans notre présente étude, nous proposons, pour le contrôle d'hygiène du lait, l'utilisation de la propriété que possèdent les bacilles du groupe Coli, ainsi que certains autres germes microbiens bacilles provenant du sol, de décomposer les nitrates en nitrites, ces microbes étant les agents principaux de la contamination du lait.

La technique de la nitroréductase (selon le nom que nous avons donné nous-mêmes à notre méthode) est basée sur la recherche, dans le lait examiné, des nitrites formés par suite de la décomposition des nitrates, le lait ayant préalablement été dilué avec une solution à $1 \%$ de nitrate de potasse et tenu à une température de $37-40^{\circ} \mathrm{C}$. dans un bain-marie ou une étuve.

Le résultat de la réaction positive causée par les nitrites, en corrélation avec la réduction plus ou moins avancée des nitrates et le temps écoulé dans l'accomplissement de cette décomposition, peuvent constituer un critérium de valeur dans la détermination du degré de pureté et réciproquement de contamination du lait.

La méthode de la nitroréductase peut être considérée comme étant plus sure, dans le contrôle d'hygiène du lait, que celle, actuellement en faveur, de la réductase, parce que son résultat positif est exclusivement dî aux agents microbiens, tandis que dans la méthode de la réductase d'autres agents peuvent également intervenir.

(Institut chimique et bactériologique, 22 , rue Socratous, Athènes.) 


\title{
BIBLIOGRAPHIE
}

[1] Code des ordonnances relatives aux aliments, boissons et objets d'usage commun (Laboratoire Général de l'Etat, Athènes, 1941).

[2] Schoenbein. Journ. F. Prakt. Chem., 1864, 62, 152.

[3] F. Roenman. Zeitschr. F. Physiol. Chem., 1881, 5, 241.

[4] Gayon et Dupetit. Comptes Rendus Acad. Sciences, 1882, 95, et autres travaux publiés dans la même Revue, 1886.

[5] P. Manil. Microbes et Actions microbiennes, Liège, 1945, 126.

[6] M. Lemoigne, R. Desveaux, R. Gavard. Comptes Rendus Acad. Sciences, 1944, 218.

\section{LE DOSAge DE LA MATIÉRE GRASSE DES CRÈmies PAR LA DÉTERMINATION DU TAUX D'HUMIDITÉ (1)}

$$
\text { par }
$$

\author{
André CAMUS, Jacqueline BURDin, Annie LE GUEN \\ (Station Centrale de Technologie Agricole, Paris)
}

Les nombreuses méthodes utilisées pour le dosage de la matière grasse des crèmes sont des méthodes acidobutyrométriques. L'accord n'est pas total sur la valeur de chacune et l'on peut dire qu'aucune ne s'est imposée d'une façon absolue à l'ensemble des utilisateurs.

Nous rappellerons tout d'abord quelques-uns des reproches qui peuvent leur être adressés :

1. Dans les meilleures conditions d'utilisation, il n'est pas possible d'apprécier dans la lecture des butyromètres plus d'une demi division. C'est dire que la matière grasse des crèmes, par ces méthodes, est mesurée, au mieux, à $0,5 \%$ près. Les écarts observés sont souvent plus grands. Nous ne tenons pas compte, dans cette approximation, des erreurs, inhérentes à toutes les méthodes, qui peuvent être commises au cours des prélèvements d'échantillons ; il s'agit là d'un autre problème qui n'a pas encore obtenu de solution satisfaisante : celui de l'homogénéité des échantillons.

2. Elles utilisent des acides concentrés dont la manipulation présente un certain danger.

3. Elles nécessitent un matériel onéreux et le prix de revient de l'analyse est assez élevé.

Nous avons pensé que la méthode simple basée sur la détermination de l'humidité de la crème présentait peut-être une précision suffisante et une simplicité plus grande.

(1) Communication présentée au VIII ${ }^{\mathrm{e}}$ Congrès International des Iredustries A gricoles, Bruxelles, 9-15 juillet 1950. 